\title{
Projeto Encontre-me! TICs para a Divulgação e Busca de Pessoas Desaparecidas de Maneira Colaborativa
}

\author{
Tadeu Classe ${ }^{1,2}$, Gildo Leonel ${ }^{2}$, Iran dos Reis Costa ${ }^{2}$, Matheus Dore ${ }^{2}$, Samuel \\ Braz $^{2}$, Camila Campos², Camillo Falcão ${ }^{2}$, Daves Martins², Miriã Correa ${ }^{2}$ \\ ${ }^{1}$ Programa de Pós-Graduação em Informática (PPGI) - Universidade Federal do Estado \\ do Rio de Janeiro (UNIRIO) \\ Rio de Janeiro - RJ - Brasil \\ ${ }^{2}$ Análise e Desenvolvimento de Sistemas / Sistemas Para Internet \\ Faculdade Integradas Vianna Júnior (FIVJ) - Juiz de Fora - MG - Brasil \\ tadeu.classe@uniriotec.br, \\ \{gleonel, cmcampos, cfalcao, dmartins, coordsi\}@vianna.edu.br, \\ \{iran.junior, matheus. dore, samuel.braz\}@viannasempre. com.br
}

\begin{abstract}
In the big cities of Brazil and the World, the people disappearance is a big problem that can reach any family independent their social class. Nevertheless, the service of search to missing people, although so much important, is unknown by the society and it does not have necessary divulgation. People, due to the rising of the social media, make posts with information about disappearing aiming to obtain some information. In that context, this word presents the research design of the project Encontre-Me!, which is based on Design Science Research Methodology. The project aims to develop and deliver ICTs and service to include, search and spread missing people, allowing that people share information about disappearing, and also, allowing them to known that important public service.
\end{abstract}

Resumo. Nas grandes cidades do Brasil e do Mundo o desaparecimento de pessoas é uma realidade que pode atingir qualquer família, independente da classe social. Contudo, o serviço de busca de pessoas desaparecidas, embora muito importante, é pouco conhecido pela sociedade e não é divulgado de forma precisa. As pessoas, devido ao crescimento das mídias sociais, realizam divulgação dos desaparecidos nestes veículos na esperança de obter informações sobre o paradeiro de seu ente querido. Neste contexto, este trabalho apresenta o desenho de pesquisa do projeto Encontre-me!, usando Design Science Research Methodology como metodologia. Este projeto objetiva desenvolver e disponibilizar TICs e serviços para inclusão busca e divulgação de pessoas desaparecidos, fazendo com que as pessoas possam colaborar na busca por informações, além de conhecer melhor este serviço tão importante.

\section{Introdução}

Os serviços públicos são criados em instituições nacionais, estaduais e locais, com o objetivo de serem entregues aos cidadãos, organizações, entidades jurídicas etc., para o exercício da cidadania, podendo ser considerado uma obrigação moral dos governos, prevista nos direitos humanos tanto quanto o direito à água, energia, comida, segurança e saúde [Bertot et al., 2016]. 
Em seu dia-a-dia, os cidadãos, na maioria das vezes, conhecem e utilizam diversos serviços públicos como, por exemplo, solicitação de guias e pagamento de contas, serviços relacionados à saúde, consultas em documentos de web sites etc. [CGI. $\mathrm{Br}, 2016]$. Muitos outros serviços públicos são desconhecidos, embora tenham importância ímpar na vida dos cidadãos. Estes serviços somente são conhecidos e requisitados em momentos de extrema necessidade. Desta forma, além das dificuldades ao prestar os serviços já enfrentadas pelas instituições públicas devido à falta de recursos, baixa transparência e gerenciamento inadequado de recursos, o desconhecimento sobre a disponibilidade e execução dos serviços fazem com que eles pareçam ineficazes aos cidadãos [Winters et al. 2014].

Um destes importantes serviços, porém, pouco conhecido pela população é o de descoberta de pessoas desaparecidas. Embora existam várias delegacias especializadas na descoberta do paradeiro de pessoas desaparecidas, este não é um serviço muito divulgado, e devido a isso, as pessoas não costumam conhecer o seu processo, como ele é solicitado e executado, fazendo que estas pessoas recorram a ajuda em meios alternativos como mídias sociais, por exemplo [Souza, 2012].

Neste contexto, este trabalho apresenta o design de pesquisa do projeto Encontre-me! Este projeto (ainda seus passos iniciais) conta com uma coleção de TICs (Tecnologias de Informação e Comunicação), e serviços para o registro, busca, divulgação e notificação de pessoas desaparecidas, de maneira colaborativa. A partir destes serviços e aplicações, o projeto Encontre-me! tem como objetivo fazer com que as pessoas com entes desaparecidos, possam divulgar, buscar e obter informações de desaparecimentos em várias frentes, como aplicativos mobile, jogos, mídias sociais, plataformas de participação, etc. Em outra mão, estas TICs buscam apresentar e permitir a conscientização da população sobre a importância do serviço de pessoas desaparecidas, permitindo a elas colaborar com informações úteis que levem a descoberta de paradeiro dos desaparecidos.

Portanto, este trabalho está organizado em: Seção 2, apresenta as definições e conceitos sobre a Design Science Research, a ser usada na condução deste projeto. A seção 3, apresenta o design deste projeto dento da metodologia selecionada. E a seção 4 apresenta as conclusões e direções futuras do projeto Encontre-Me!

\section{Design Science Research}

A Design Science Research (DSR) é uma abordagem epistemológica que operacionaliza a investigação fundamentada no paradigma da design science [Dresch et al., 2015]. Tal abordagem epistemológica vem se popularizando na área de Sistemas de Informação (SI), na qual as pesquisas objetivam a produção de conhecimento que possa ser utilizado no desenvolvimento de soluções tecnológicas para diversos tipos de problemas [Hevner et al., 2004]. Na DSR, os artefatos são projetados com base em conjecturas, sendo que a avaliação destes artefatos visa averiguar a validade de tais conjecturas, contribuindo com o conhecimento científico e tecnológico [Rocha et al., 2015].

De acordo com Peffers et al. (2007), existem diversas pesquisas que buscam trazer a design science para a comunidade de Sistemas de Informação, tendo sucesso em criar casos que tragam validade e valores da DSR para suas pesquisas. Para conduzir as pesquisas em DSR é necessário um framework aceitável e comum que permita aos pesquisadores reconhecer e avaliar os resultados. Pensando nisso esses autores propuseram o Design Science Research Methodology (DSRM), um método de pesquisa em DSR para pesquisas em SI. 
A construção da metodologia DSRM buscou na literatura sobre design science pesquisas anteriores, identificando os elementos chave que deveriam, ou não, ser incluídos. Desta maneira, o método foi criado considerando os elementos mais aceitos e utilizados pelos pesquisadores, os quais, de acordo com Peffers et al. (2007) são: i) Identificação do Problema e Motivação: definição do problema de pesquisa e a sua justificativa; ii) Definição de Objetivos para a Solução: inferência sobre as conjecturas e objetivos para solução a partir da definição e conhecimento sobre o problema; iii) Design e Desenvolvimento: criação do artefato para tentar satisfazer o problema; iv) Demonstração: demonstração de que o artefato proposto pode satisfazer um ou mais instâncias do problema; v) Avaliações: observar e mediar o quão bem o artefato suporta a solução do problema, comparando seus objetivos com os resultados a partir do uso do artefato na demonstração. É comum na avaliação o uso de pesquisas qualitativas e/ou quantitativas, as quais buscam evidenciar os objetivos e conjecturas inferidas na etapa de definição dos objetivos; e, vi) Comunicação: comunicação sobre o problema e sua importância, o artefato e as inovações que ele trouxe o rigor de seu design, e o conhecimento gerado.

\section{Design da Pesquisa - O Projeto Encontre-Me!}

Como neste projeto de pesquisa espera-se o design, reflexão e construção de artefatos tecnológicos que contribuam na divulgação e colaboração sobre informações de pessoas desaparecidas, bem como a compreensão do cidadão sobre a importância deste serviço, o uso do DSRM parede adequado.

Desta forma, dentre os vários possíveis pontos de entrada do DSRM, este trabalho teve como marco inicial o desenvolvimento de um projeto centrado no seguinte objetivo: Desenvolver uma plataforma de serviços e tecnologias de informação e comunicação capazes de auxiliar na inclusão busca divulgação e notificação de pessoas desaparecidas de forma colaborativa e fazendo com que as pessoas tomem conhecimento da importância e funcionamento do serviço de descoberta de paradeiros.

Considerando os elementos da DSRM, é possível descrever este projeto de pesquisa como: para atender ao objetivo apresentado, é considerado como contexto 0 serviço de busca e descoberta de pessoas desaparecidos (descoberta de paradeiros), sendo proposto como artefatos para alcançar esse objetivo o desenvolvimento de serviços e TICs que auxiliem na inclusão, busca, divulgação e notificação de pessoas desaparecidas de maneira colaborativa, auxiliando na divulgação e reflexão sobre sua importância. Tendo como apoio ao desenvolvimento destes artefatos, as bases teóricas os arcabouços conceituais e definições advindos de democracia digital, participação social e engajamento; além das bases técnicas voltadas aos princípios de arquiteturas orientadas a serviços (SOA), design de jogos digitais, desenvolvimento de aplicativos móveis e web sites.

Os serviços e TICs propostos como artefatos desta pesquisa visam validar as conjecturas teóricas de que: i) é possível construir uma plataforma de serviços e aplicações que auxiliem na publicação e divulgação de pessoas desaparecidas de forma colaborativa; ii) a arquitetura de serviços aliada a uma base de dados única possibilita o uso de informações de desaparecidos em vários sistemas diferentes, contribuindo com a disseminação de informações; iii) a criação de jogo digital com as informações reais de desaparecidos pode contribuir com sua divulgação, permitindo que o jogador tome conhecimento de pessoas desaparecidas reais, e do funcionamento do serviço público; iv) os artefatos desenvolvidos contribuirão com o serviço de busca e notificação de pessoas desaparecidas, permitindo interação entre pessoas de maneira colaborativa, além da divulgação de informação em mídias sociais. 
Como avaliação dos artefatos: i) demonstração das aplicações do projeto Encontre-Me! através de provas de conceito, estudos de caso e observações de uso dos artefatos; ii) avaliação de usabilidade e utilidade das TICs com cidadãos; iii) realização de estudo de caso a fim de averiguar se as soluções desenvolvidas no projeto EncontreMe! foram capazes de divulgar e captar informações de pessoas desaparecidas e contribuir com a reflexão social sobre a importância deste serviço público.

\subsection{Contexto e Problema}

O desaparecimento de pessoas é uma realidade presente nas grandes cidades do mundo, podendo acontecer com qualquer família, independentemente de seu contexto e classe social. No Brasil, de acordo com o Fórum Brasileiro de Segurança Pública, considerando os anos de 2007 a 2017, ocorreram cerca de 8 desaparecimentos de pessoas por hora [Acayaba, 2017], em um total de 786.071 desaparecidos neste intervalo de tempo, sendo 82 mil desaparecidos foram registrados entre 2016 e 2017. Somente no Estado do Rio de Janeiro, considerando os dados publicados em julho de 2017, no período de 15 anos (2012 a 2017), foram comunicados à polícia civil, aproximadamente 33 mil ocorrências [Grandin E Coelho, 2017]. Em São Paulo, cerca de 25 mil pessoas desapareceram no ano de 2017 segundo Ministério Público paulista [Sacheto, 2019].

No Brasil, existem várias delegacias especializadas na descoberta do paradeiro de pessoas desaparecidas, entretanto estes departamentos não possuem recursos suficientes para manterem suas bases de dados atualizadas, e realizarem a melhor divulgação do desaparecimento. Outro problema comum enfrentado na prestação deste serviço é que as pessoas não sabem como solicitá-lo ou muitas vezes tem receio de fazê-lo [Souza, 2012], optando, em muitos dos casos, por divulgações informações do desaparecimento em mídias sociais.

\subsection{Trabalhos Relacionados}

Existem vários SIs para divulgação e busca de pessoas desaparecidas, dentre eles uma importante iniciativa para registrar os desaparecimentos foi o banco de dados de pessoas desaparecidas mantido pelo Mistério da Justiça, contudo um dos seus desafios é manter estas informações atualizadas [Ferreira et al., 2018]. Ferreira et al. (2018), apresentaram um sistema de informação baseado na recuperação de informação de vários sites e portais na internet, agregando estas informações em uma única aplicação. Além disso, o sistema consegue analisar dados, agrupando informações e gerando conhecimento útil sobre pessoas desaparecidas.

Além das buscas, até mesmo a divulgação de uma pessoa desaparecida às vezes não é uma tarefa simples. Classe et al. (2017a), a partir de uma parceria com a delegacia de descoberta de paradeiros (DDPA) da Polícia Civil do Rio de Janeiro, desenvolveram um aplicativo mobile para consultas da base de dados de desaparecidos e divulgação de cartazes (uma pequena imagem informativa com dados de desaparecido) em mídias sociais.

Sobre a apresentação e divulgação do serviço de descoberta de paradeiro para a sociedade, Classe et al. (2017b) desenvolveram o jogo digital Desaparecidos (http://tadeuclasse.com.br/games/desaparecidos), no intuito de apresentar o serviço à sociedade, facilitando a sua compreensão.

\subsection{Artefatos Propostos}

A principal proposta desta pesquisa para atingir ao objetivo especificado é a construção de serviços e aplicações que auxiliem na divulgação e busca de informações de pessoas 
desaparecidas de maneira colaborativa e contribua com que a sociedade conheça e existência e importância deste serviço.

Neste sentido o projeto Econtre-Me! surgiu da ideia de criar este arcabouço tecnológico, baseado em serviços conectados a uma base de informação única, que podem ser acessados por diferentes aplicações. Em termos tecnológicos o projeto Encontre-me! é bem simples. Ele é formado, principalmente de uma API de serviços web e alguns sistemas de informações que fazem uso desta API no sentido de manter e consumir os dados existentes nesta base de dados única (Figura 1).

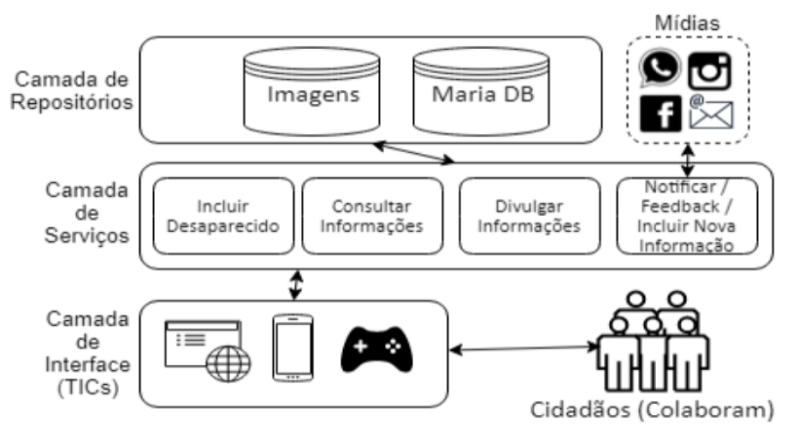

Figura 1 - Arquitetura do Projeto Encontre-Me!

Inicialmente para acesso do cidadão, o projeto Encontre-Me! tem a API de serviços como núcleo e é circundado pôr em três sistemas principais, de acordo com a Tabela 1.

\section{Tabela 2 - Sistemas do Projeto Encontre-Me!}

\begin{tabular}{|l|l|}
\hline Sistema & Descrição \\
\hline $\begin{array}{l}\text { Portal } \\
\text { Me! }\end{array}$ & $\begin{array}{l}\text { Este portal é uma peça fundamental do projeto, pois permite que sejam cadastradas informações de } \\
\text { pessoas desaparecidas e divulgadas estas informações em diversos veículos de comunicação como } \\
\text { mídias sociais, e-mail etc. Ao cadastrar informações de desaparecimento, o responsável deve aceitar } \\
\text { um termo de veracidade de informações, além do termo que as pessoas responsáveis pelo projeto } \\
\text { Encontre-Me! Não possuem responsabilidade sobre as informações declaradas e divulgadas. Este } \\
\text { portal também disponibiliza ferramentas de buscas de pessoas desaparecidas, e ainda a permite a } \\
\text { colaboração de outros usuários do sistema, ao notificar os responsáveis com informaços sobre a } \\
\text { pessoa desaparecida. Embora os responsáveis do projeto não tenham responsabilidade sobre as } \\
\text { informações, existe uma equipe de verificação de notificações, mensagens etc., a qual busca, ao } \\
\text { máximo, filtrar possíveis informações que não tenham a ver com o contexto daquele } \\
\text { desaparecimento. }\end{array}$ \\
\hline $\begin{array}{l}\text { Encontre- } \\
\text { Me! Mobile }\end{array}$ & $\begin{array}{l}\text { O aplicativo é um sistema simples no qual o seu usuário pode realizar busca de pessoas } \\
\text { desaparecidas, divulgar informações em mídias sociais e colaborar com notificações e informações } \\
\text { de desaparecimento. }\end{array}$ \\
\hline $\begin{array}{l}\text { Encontre- } \\
\text { Me! Game }\end{array}$ & $\begin{array}{l}\text { O jogo é desenvolvido, basicamente, para divulgação de pessoas desaparecidas de uma maneira } \\
\text { interessante para o cidadão. Além disso, o objetivo aqui é que o cidadão aprenda o serviço público } \\
\text { de descoberta de desaparecidos, como é realizado, quais suas ferramentas etc. Além disso, o jogo } \\
\text { possui meios de divulgar desaparecidos em mídias sociais. }\end{array}$ \\
\hline
\end{tabular}

Além destes sistemas vinculados ao projeto Encontre-Me!, a API de serviços estará disponível para uso por qualquer outro sistema, possibilitando que sejam integrados diferentes fontes de informações, colaborando ainda mais com a descoberta de informações de pessoas desaparecidas e a divulgação de informações de utilidade pública para a sociedade.

\section{Conclusões e Trabalhos Futuros}

Este artigo teve como objetivo apresentar o design da pesquisa do projeto EncontreMe!, o qual visa desenvolver e construir serviços e TICs para auxiliar na busca, divulgação e notificação de pessoas desaparecidas colaborativamente, além de conscientizar à sociedade sobre a existência e importância deste serviço. 
Como o objetivo desde projeto é o de projetar e entregar sistemas de informação para o contexto do serviço de busca de desaparecidos, este trabalho será desenvolvido usando a Design Science Research Methodology, buscando averiguar as conjecturas teóricas estabelecidas, baseando-se em suas referências científicas e tecnológicas. E, desta maneira, contribuir com a sociedade na busca de pessoas desaparecidas.

\section{Referências}

Acayaba, B. (2017). "Brasil registra 8 desaparecimentos por hora nos últimos 10 anos, diz estudo inédito". Portal de Notícias G1.

Bertot, J.; Estevez, E.; Janowski, T. (2016). "Universal and contextualized public services: Digital public service innovation framework". In: Government Information Quarterly, v.33(2), pp. 211-222.

CGI.Br. (2016). "ICT Electronic Government 2015: Survey on the Use of Information and Communication Technologies in the Brazilian Public Sector". Brazilian Internet Steering Committee.

Classe, T.M.; Araujo, R.M.; Lima, V.R.; Ferreira, H.A.G. (2017a). "Desaparecidos RJ Um Sistema de Informação Para Apoio à Busca de Pessoas Desaparecidas no Estado do Rio de Janeiro". In: Escola Regional de Sistemas de Informação do Rio de Janeiro.

Classe, T., Araujo, R., \& Xexeo, G. (2017b). Desaparecidos RJ - Um Jogo Digital para o Entendimento de Processos de Prestação de Serviços Públicos. XVI Simpósio Brasileiro de Jogos e Entretenimento Digital (SBGames 2017). Curitiba.

Dresch, A.; Lacerda, D.P.; Antunes, J.A.V. (2015). "Design Science Research: método de pesquisa para avanço da ciência e tecnologia". Porto Alegre, Bookman.

Ferreira, R.S.; Oliveira, C.G.; Lima, A.A.B. (2018). Myosotis: An Information System Applied to Missing People Problem. In: Simpósio Brasileiro de Sistemas de Informação.

Grandin, F., \& Coelho, H. (2017). "Rio tem 33 mil desaparecidos em 15 anos; Zona Oeste e Bonsucesso concentram casos". Portal de Notícias do G1.

Hevner, A.; March, S.; Park, J.; Ram, S. (2004). "Design Science in Information Systems Research". In: MIS Quarterly, v.28(1), pp.75-105.

Peffers, K.; Tuunanen, T.; Rothenberger M.A.; Chatterjee, S. (2007). "A design science research methodology for information systems research". In: Journal of Management Information Systems (JMIS), v.24(3), pp. 45-77.

Rocha, E. B.; Pimentel, M.; Diniz, M.C., Santoro, F.M. (2015). "Design Science Research para o Desenvolvimento de um Modelo da Participação em Bate-papo". In: iSys-Revista Brasileira de Sistemas de Informação, v.8(1), pp. 18-41.

Sacheto, C. (2019). "Nos últimos 2 anos, mais de 82 mil pessoas desapareceram no Brasil”. Portal de Notícias R7.

Souza G.M. (2012). "Questões Sociais Envolvidas e Perfil dos Desaparecidos na Capital do Estado do Rio de Janeiro em 2010”. Ph.D. Dissertation. Universidade Cândido Mendes.

Winters, M.; Karim, A.G.; Martawardaya, B. (2014). "Public Service Provision under Conditions of Insufficient Citizen Demand: Insights from the Urban Sanitation Sector in Indonesia". In: World development, v.60, pp. 31-42. 